\title{
Obber die Ausscheidungsgröße per os, subkutan und intravenös eingeführter Harnsäure beim Kaninchen.
}

\author{
Von
}

Ernst Bendix und Alfred Schittenhelm.

\author{
(Aus dem Laboratorium der Göttinger medizinischen Klinik.) \\ (Der Redaktion zugegangen am 21. Juli 1904.)
}

Gelegentlich anderer Untersuchungen war es für uns von Wert, festzustellen, wie sich die Ausscheidung der per os, subkutan und intravenös eingeführten Harnsäure beim Kaninchen gestaltet.

Unsere Versuchsanordnung war folgende:

Möglichst gleichartig ernährten Kaninchen wurde in Piperazin gelöste Harnsäure der Reihe nach intravenös, subkutan und per os beigebracht. Wir haben Versuchsreihen von einund zweitägigen Perioden gewählt, mit entsprechend langen Intervallen. Auf die Wiedergabe vom Gesamt-N-Werte verzichten wir, da nach unseren Erfahrungen derselbe zu großen Schwankungen unterliegt, um für die Beurteilung der resorbierten Harnsäuremenge von wesentlicher Bedeutung zu sein. Die Abgrenzung des Urins geschah durch manuelles Ausdrücken, was bei einiger Übung durchaus genügt. Die Methoden waren die gebräuchlichen. Es bedarf jedoch besonderer Bemerkung, daß der sedimentreiche Kaninchenharn zunächst mit Natronlauge gekocht und heiß filtriert wurde. Außerdem hat uns zur Klärung des Filtrats vom Schwefelsilber eine Beigabe von kleinen Mengen ges. Aluminiumacetatlösung (Wiener) gute Dienste geleistet.

Versuch I.

Kräftiges weibliches Kaninchen, $2190 \mathrm{~g}$ Gewicht. 
Tabelle I.

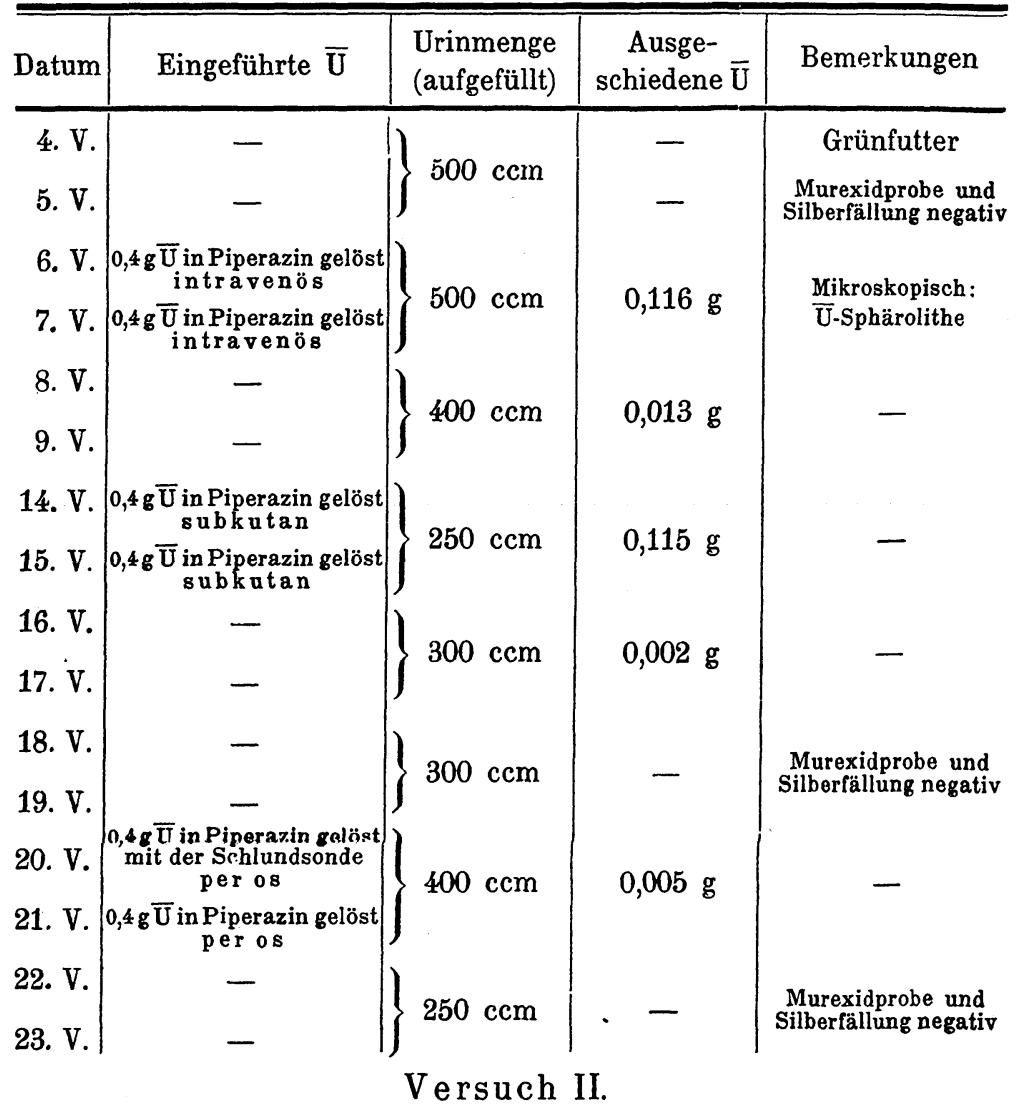

Kräftiges, gesundes Kaninchen, weiblich, 2990 g schwer. Tabelle II.

\begin{tabular}{|c|c|c|c|c|}
\hline Datum & Eingeführte $\overline{\mathrm{U}}$ & $\begin{array}{l}\text { Urinmenge } \\
\text { (aufgefüllt) }\end{array}$ & $\begin{array}{c}\text { Ausge- } \\
\text { schiedene } \bar{U}\end{array}$ & Bemerkungen \\
\hline $\begin{array}{l}\text { 8. VI. } \\
\text { 9. VI. }\end{array}$ & $\mid \begin{array}{c}0,5 \mathrm{~g} \overline{\mathrm{U}} \text { in Piperazin gelöst } \\
(20 \mathrm{ccm}) \text { in tra v eno } \mathrm{s} \\
-\end{array}$ & $300 \mathrm{ccm}$ & $0,044 \mathrm{~g}$ & Grünfutter \\
\hline $\begin{array}{l}\text { 10. VI. } \\
\text { 11. VI. }\end{array}$ & $\mid \begin{array}{c}0,5 \mathrm{~g} \overline{\mathrm{U}} \text { in Piperazin gelöst } \\
(20 \mathrm{ccm}) \text { s u b k u t a n } \\
-\end{array}$ & $325 \mathrm{ccm}$ & $0,022 \mathrm{~g}$ & $\Rightarrow$ \\
\hline $\begin{array}{l}13 . \mathrm{VI} . \\
14 . \mathrm{VI} .\end{array}$ & $\left|\begin{array}{c}0,5 \mathrm{~g} \overline{\mathrm{U}} \text { in Piperazin gelöst } \\
(20 \mathrm{ccm}) \\
\text { mit Schlundsonde per os } \\
-\end{array}\right|$ & $350 \mathrm{ccm}$ & $\underset{\text { nachweisbar }}{\text { Keine } \overline{\mathrm{U}}}$ & $>$ \\
\hline
\end{tabular}


Versuch III.

Ein gesundes Kaninchen erhält am 28. VI. 0,5 g Harnsäure in $15 \mathrm{ccm}$ Wasser mit Piperazinzusatz bis zur Lösung intravenös (Ohrvene).

Die Urinmenge der nächsten 48 Stunden betrug ca. $350 \mathrm{ccm}$. Harnsäure $=0,056 \mathrm{~g}$.

\section{Versuch IV.}

Ein normales Kaninchen erhält am 4. VII. 0,5 g Harnsäure ebenso gelöst subkutan. Die Urinmenge der nächsten 48 Stunden beträgt ca. $270 \mathrm{ccm}$. Harnsäuremenge $=\mathbf{0 , 0 8 7 4} \mathrm{g}$.

Aus vorstehenden Versuchen ergibt sich, daß im allgemeinen die größte Menge Harnsäure im Urin wiedererscheint bei intravenöser Verabfolgung; von der per os eingegebenen Harnsäure werden nur Spuren als solche ausgeschieden; etwa in der Mitte steht die Harnsäurezufuhr bei subkutaner Verabreichung. Aber auch die höchste Harnsäureausscheidung ist doch im Vergleich zur eingeführten Menge nur eine sehr geringe, was um so bemerkenswerter ist, wenn man sich die mikroskopischen Bilder ${ }^{1}$ ) der Nieren bei derartigen Versuchen vergegenwärtigt.

Unsere Befunde beanspruchen insofern ein weitergehendes Interesse, als sie geeignet scheinen, manche widersprechende Versuche der Purinharnsäureliteratur zu erklären. Wenn günstigstenfalls $18 \%$ der einverleibten Harnsäure als solche im Harn wiedererscheint, so ist es verständlich, daß bei Darreichung per os selbst verhältnismäßig große Mengen von Purinkörpern auf die Harnsäureausscheidung keinen nennenswerten Einfluß hervorzubringen brauchen. Jedenfalls sind Versuche mit Dezigrammen von Purinkörpern, wie sie sich in der Literatur finden, völlig unbeweisend, sogar vollkommene Resorption vorausgesetzt.

1) Ebstein u. Nikolaier bezw. Ebstein u. Bendix, Virchows Archiv, Bd. 134 resp. 178.

Hoppe-Seyler's Zeitschrift f. physiol. Chemie. XLII. 